\title{
CORRECTION
}

\section{Author Correction: Delivery of acetylthevetin B, an antitumor cardiac glycoside, using polymeric micelles for enhanced therapeutic efficacy against lung cancer cells}

Jing-jing Zhu ${ }^{1,2}$, Xin-xin Zhang ${ }^{2}$, Yun-qiu Miao ${ }^{2}$, Shu-fang He ${ }^{2}$, Dan-mei Tian ${ }^{1}$, Xin-sheng Yao ${ }^{1}$, Jin-shan Tang ${ }^{1,3}$ and Yong Gan ${ }^{2}$ Acta Pharmacologica Sinica (2020) 41:1261; https://doi.org/10.1038/s41401-020-0362-1

Correction to: Acta Pharmacologica Sinica https://doi.org/10.1038/ aps.2016.113, published online 05 December 2016.

After publication, the authors realized that the representative images of DIR group in Fig. 4a were found to be misplaced due to the mishandling in the manuscript preparation. All the authors agree on the correction of our negligence as providing the corrected Fig. 4a presented below. This correction does not affect the results and conclusion of the paper. We sincerely apologize for our mistakes and any inconvenience this might have caused.

a Control 15min $6 \mathrm{~h}$ Epi-fluorescence

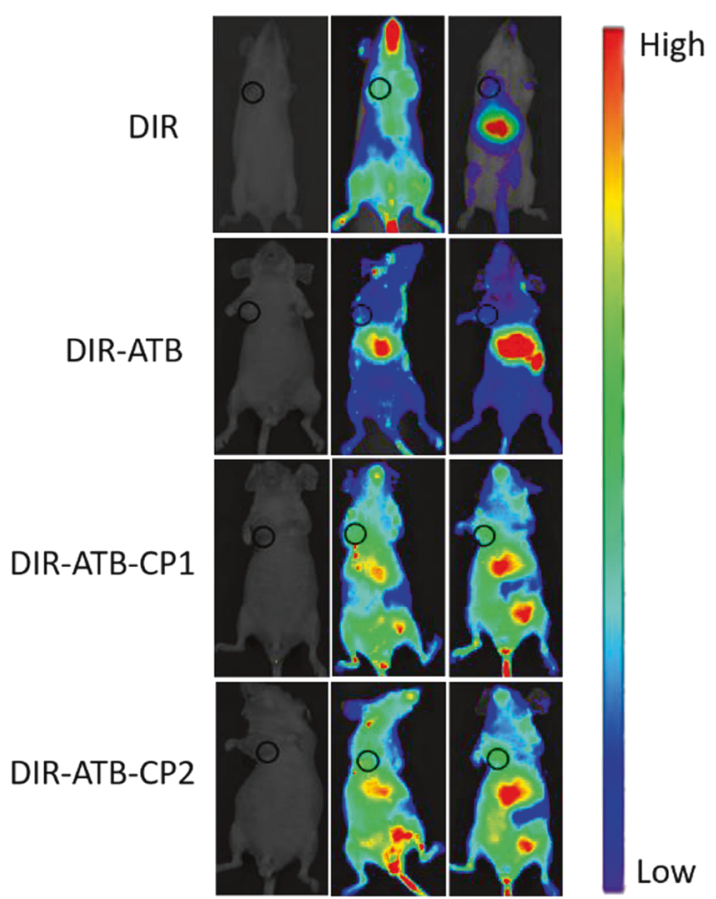

Fig. 4 Biodistribution of $1,1^{\prime}$-dioctadecyl-3,3,3', $3^{\prime}$-tetramethylindotricarbocyanine iodide (DIR)-labeled formulations after intravenous (iv) injection in mice. a Real-time in vivo fluorescence images of mice after iv injection of free DIR, DIR labeled acetylthevetin B (ATB) solution (DIR-ATB), DIR-labeled CP1(DIR-ATB-CP1), and DIR-labeled CP2 (DIR-ATB-CP2) (lex = $745 \mathrm{~nm}$; lem = $800 \mathrm{~nm}$ ).

${ }^{1}$ Institute of Traditional Chinese Medicine and Natural Products, College of Pharmacy, Jinan University, Guangzhou 510632, China; ${ }^{2}$ Shanghai Institute of Materia Medica, Chinese

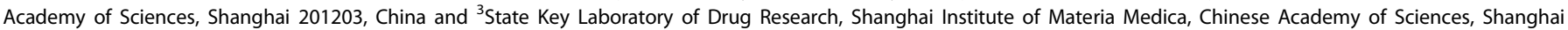
201203, China

Correspondence: Xin-xin Zhang (xinxinzhang@simm.ac.cn) or Jin-shan Tang (gztangjinshan@126.com) or Yong Gan (ygan@simm.ac.cn)

Published online: 21 February 2020 